\title{
A feasibility study testing four hypotheses with phase II outcomes in advanced colorectal cancer (MRC FOCUS3): a model for randomised controlled trials in the era of personalised medicine?
}

T S Maughan ${ }^{1}$, A M Meade ${ }^{*}, 2$ R A Adams ${ }^{3}$, S D Richman ${ }^{4}$, R Butler $^{5}$, D Fisher ${ }^{2}$, R H Wilson ${ }^{6}$, B Jasani $^{7}$, G R Taylor ${ }^{4}$, G T Williams ${ }^{7}$, J R Sampson ${ }^{7}$, M T Seymour ${ }^{8}$, L L Nichols ${ }^{2}$, S L Kenny ${ }^{2}$, A Nelson ${ }^{9}$, C M Sampson ${ }^{9}$, E Hodgkinson $^{10}$, J A Bridgewater ${ }^{11}$, D L Furniss ${ }^{10}$, R Roy ${ }^{12}, \mathrm{M}$ J Pope ${ }^{2,13}$, J K Pope ${ }^{2,13}$, M Parmar ${ }^{2}$, P Quirke ${ }^{4}$ and R Kaplan ${ }^{2}$

${ }^{1}$ CRUK/MRC Oxford Institute for Radiation Oncology, University of Oxford, Oxford OX3 7DQ, UK; ${ }^{2} M R C$ Clinical Trials Unit at UCL, Institute of Clinical Trials and Methodology, London WC2B 6NH, UK; ${ }^{3}$ Cardiff University and Velindre Cancer Centre, Cardiff, UK; ${ }^{4}$ Leeds Institute of Cancer and Pathology, University of Leeds, Leeds LS9 7TF, UK; ${ }^{5}$ University Hospital of Wales, Cardiff CF14 4XW, UK; ${ }^{6}$ Centre for Cancer Research and Cell Biology, Queen's University Belfast, Belfast BT9 7AE, UK; ${ }^{7}$ Institute of Cancer and Genetics, Cardiff University, Cardiff CF14 4XN, UK; ${ }^{8}$ St James's Institute of Oncology, University of Leeds, Leeds LS9 7TF, UK; ${ }^{9}$ Wales Cancer Trials Unit, Cardiff University, Cardiff CF14 4YS, UK; ${ }^{10}$ Sheffield Teaching Hospitals NHS Foundation Trust, Sheffield S5 7AU, UK; ${ }^{11}$ UCL Cancer Institute, London WC1E 6BT, UK and ${ }^{12}$ Department of Oncology, Castle Hill Hospital, East Riding of Yorkshire HU16 5JQ, UK

\footnotetext{
Background: Molecular characteristics of cancer vary between individuals. In future, most trials will require assessment of biomarkers to allocate patients into enriched populations in which targeted therapies are more likely to be effective. The MRC FOCUS3 trial is a feasibility study to assess key elements in the planning of such studies.

Patients and Methods: Patients with advanced colorectal cancer were registered from 24 centres between February 2010 and April 2011. With their consent, patients' tumour samples were analysed for KRAS/BRAF oncogene mutation status and topoisomerase 1 (topo-1) immunohistochemistry. Patients were then classified into one of four molecular strata; within each strata patients were randomised to one of two hypothesis-driven experimental therapies or a common control arm (FOLFIRI chemotherapy). A 4-stage suite of patient information sheets (PISs) was developed to avoid patient overload.
}

Results: A total of 332 patients were registered, 244 randomised. Among randomised patients, biomarker results were provided within 10 working days (w.d.) in $71 \%, 15$ w.d. in $91 \%$ and 20 w.d. in $99 \%$. DNA mutation analysis was $100 \%$ concordant between two laboratories. Over $90 \%$ of participants reported excellent understanding of all aspects of the trial. In this randomised phase II setting, omission of irinotecan in the low topo-1 group was associated with increased response rate and addition of cetuximab in the KRAS, BRAF wild-type cohort was associated with longer progression-free survival.

Conclusions: Patient samples can be collected and analysed within workable time frames and with reproducible mutation results. Complex multiarm designs are acceptable to patients with good PIS. Randomisation within each cohort provides outcome data that can inform clinical practice.

*Correspondence: Dr AM Meade; E-mail: a.meade@ucl.ac.uk

${ }^{13} \mathrm{Malcolm}$ and Janet Pope are Consumer Representatives; they also represent Velindre Hospital, Patient Liaison Group, Cardiff CF14 2TL, UK

Received 21 November 2013; revised 27 February 2014; accepted 13 March 2014; published online 17 April 2014

(c) 2014 Cancer Research UK. All rights reserved 0007-0920/14 
Cancer is the product of a somatic evolutionary process, in which successive advantageous genetic and epigenetic alterations drive the progression of the disease (Greaves and Maley, 2012). Although current knowledge indicates many similar changes in different cancers, the number of possible combinations of changes even within a given anatomical/histological type such as colorectal cancer (CRC) is very large (The Cancer Genome Network Atlas, 2012). This raises a major challenge in the search for effective therapies that target the properties of any given cancer, especially for advanced disease where clonal evolution and the selective pressure of prior therapies drive increasing diversity and resistance to subsequent therapy (Sequist et al, 2011; Gerlinger et al, 2012). This emerging understanding of the heterogeneity of cancer is a major challenge to clinical trialists and demands new methodologies for testing novel therapies.

Fundamental to this challenge is the identification of biomarkers that help enrich the evaluated population for benefit from a specific therapy. In CRC, the use of epidermal growth factor receptor (EGFR)-targeted therapy has led to the discovery of the importance of KRAS and recently NRAS mutations (Douillard et al, 2013) in prediction of lack of response to that therapy and association of $B R A F$ mutation with a particularly poor prognosis in advanced CRC (ACRC; Lievre et al, 2006; Karapetis et al, 2008; Maughan et al, 2011). Further biomarker candidates under evaluation as potentially predicting lack of benefit from anti-EGFR therapy are PI3K mutations and loss of PTEN expression (De Roock et al, 2010; Seymour et al, 2013).

This paper reports the results of the MRC FOCUS3 trial (ISRCTN83171665), a randomised feasibility trial for the selection of therapy for patients with ACRC based on their KRAS and BRAF mutation status as well as their topoisomerase 1 (topo-1) expression status.

\section{MATERIALS AND METHODS}

Trial design. Patients were registered on the day they provided written consent for the release of a tumour sample. Upon determination of their biomarker status, patients were allocated to one of four molecular subgroups for randomisation: (1) low topo-1 expression levels and both KRAS and BRAF wild type, (2) low topo-1 and either KRAS- or BRAF-activating mutations, (3) high topo-1 and both KRAS and BRAF wild type and (4) high topo- 1 and either KRAS or BRAF mutations. These randomisation subgroups correspond to the prior hypotheses that: (1) in patients with low topo-1 tumours, FU alone is similarly effective and therefore preferable to irinotecan/FU combination (Braun et al, 2008); (2) in patients with KRAS/BRAF wild-type tumours, anti-EGFR therapy improves outcomes (Van Cutsem et al, 2009); (3) in patients with high topo-1 tumours, addition of oxaliplatin to irinotecan/FU improves outcomes (Braun et al, 2008) and (4) in patients with KRAS/BRAF-mutated tumours, anti-VEGF therapy might improve outcomes. There was no specific rationale for a biologically targeted therapy in patients with KRAS mutations; however, there were data suggesting benefit of bevacizumab (Ince et al, 2005).

Patients were randomised centrally by the MRC CTU via telephone using minimisation and allocated in a $1: 1: 1$ ratio to the control arm (A) common to each of the four subgroups or one of two experimental regimens (Figures 1 and 2). If either molecular test failed, patients could still be randomised in a $1: 1$ ratio based on the results available (Figure 1). Treatment allocation was not masked. Randomisation was stratified by standard clinical prognostic factors.

Patients. Eligibility criteria were age $\geqslant 18$ years, colorectal adenocarcinoma, inoperable metastatic or locoregional RECIST measurable disease, no previous chemotherapy for metastases, WHO performance status 0-2 and good organ function (Maughan and Meade, 2010). Written informed consent for both molecular testing and randomisation was required.

Outcome measures and sample size. The primary outcome measures for FOCUS3 were process outcomes, namely, in this national multi-site setting, how frequently the target could be met of $\leqslant 10$ w.d. between the date of registration and: (1) the provision of results to the investigator and (2) randomisation.

The target sample size was 240 patients; if $>226$ tumour blocks were processed within 10 w.d., we could reliably state that $\geqslant 90 \%$ samples could be analysed within that time frame. If $<206$ blocks were processed within 10 w.d., we could reliably exclude a

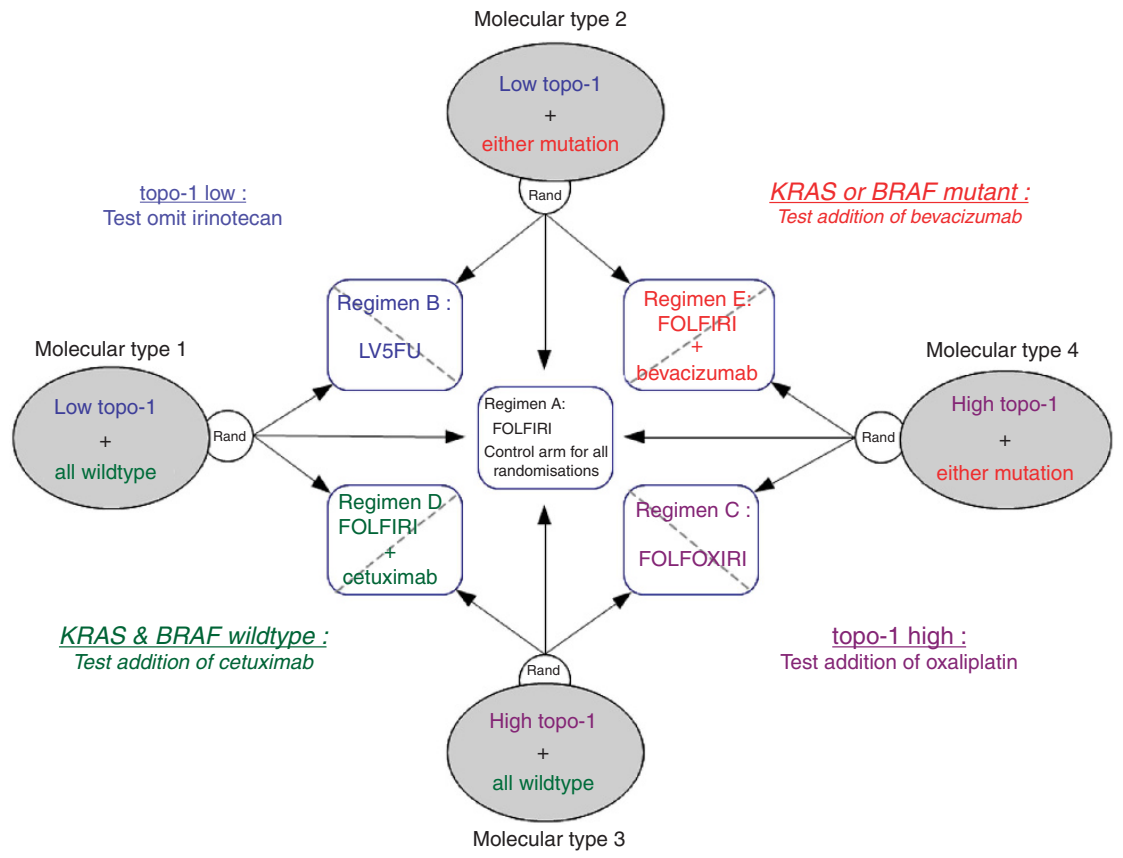

Figure 1. Trial design. 
turnaround rate of $90 \%$ (i.e., the upper $95 \%$ confidence limit would exclude $90 \%$ ).

Secondary outcome measures included toxicity, response rates (RRs) and progression-free survival (PFS) of the different regimens within each molecular subgroup; reproducibility of biomarker results and attitudes of patients to the study design, the consent process and refusal rates for trial entry.

Informed consent and patients attitudes to the trial design. A staged set of patient information sheets (PISs) was developed with input from patients, carers and nursing staff: PIS 1 explained the need for further analyses of tumour tissue using a very simple diagram and no technical details (see Figure 2), PIS2, given to patients before results of their molecular tests were known, covered the general issues of a three arm RCT and treatment side-effects. PIS3, in four specific versions a-d, describing the three arm randomisation for each of the four molecular sub-types (1-4) was given to patients before randomisation. PIS4, versions a-e, contained full details of the five treatment regimens (A-E).

Patient understanding of the information was captured on a questionnaire delivered immediately following their reading of the stage 2 PIS.

Attitudes of participants to trial entry, understanding and experience, particularly to the proposed 2 weeks time for tumour testing before treatment allocation, were evaluated by one-to-one semi-structured interviews using interpretative phenomenological analysis in a subgroup of randomised patients (Smith and Osborn, 2003).

Sample collection and analysis process. The clinical research nurse (CRN) at the recruiting hospital requested the patients' diagnostic FFPE block. Histopathology agreements were in place between MRC and all diagnostic hospitals outlining the trial rationale and stressing the importance of sending blocks promptly

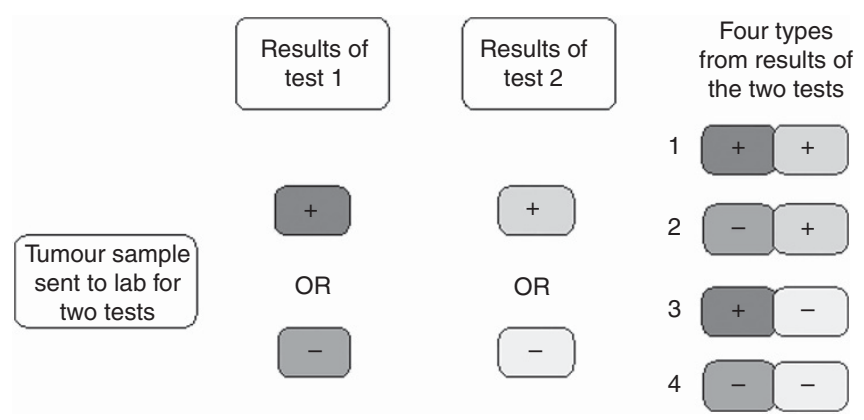

Figure 2. Diagram in patient information sheet 1 - given to patients to explain the tests carried out on their tumour sample. to the central laboratories. The MRC CTU team actively tracked samples throughout the biomarker analysis process. Upon reconfirmation of eligibility, and with their consent, patients were randomised.

Biomarker analysis. Analysis of DNA extracted from macrodissected FFPE sections of KRAS codons 12, 13 and 61 and BRAF codon 600 was each performed by Pyrosequencing (details in Supplementary Appendix).

Topo-1 protein expression was identified using a topo- 1 antibody (NCL-TOPO1; Leica, Wetzlar, Germany; details in Supplementary Appendix). Each case was scored on the basis of the percentage of positive tumour cells ( $<10 \%$ scored low, $>10 \%$ high).

Quality assurance of biomarker analysis. Fifty samples were blinded and exchanged between the two laboratories before the trial and analysed for KRAS and BRAF mutation status. Throughout the trial both laboratories took part in external quality assessment (UK NEQAS) for KRAS. Topo-1 IHC was compared between laboratories.

Interventions and assessments. The five treatment regimens were all based on the 2-weekly FOLFIRI regimen - folinic acid and irinotecan followed by bolus and infusional 5-fluouracil (5-FU; Douillard et al, 2000): (A) Control: FOLFIRI, (B) omits irinotecan: LV5FU2, (C) adds oxaliplatin: FOLFOXIRI (FOLFIRI and oxaliplatin), (D) FOLFIRI plus cetuximab and (E) FOLFIRI plus bevacizumab. Doses in (C) were dependent on patient age and WHO performance status. The chemotherapy regimens FOLFIRI and LV5FU2 are internationally recognised acronyms. The actual regimens used in FOCUS3 were established in the UK (Cheeseman et al, 2002; Leonard et al, 2002). They have been used in large numbers of patients, have been shown to be both efficacious and safe (Seymour et al, 2007) and will be referred to as FOLFIRI and LV5FU2 in this paper. The FOLFIRI regimen consisted of an IV infusion of $180 \mathrm{mg} \mathrm{m}^{-2}$ IV infusion over $30 \mathrm{~min}$ followed by $350 \mathrm{mg}$ IV infusion d,l-folinic acid or $175 \mathrm{mg}$ l-folinic acid over $2 \mathrm{~h}$. A $400 \mathrm{mg} \mathrm{m}^{-2} \mathrm{IV}$ bolus injection of 5 -FU was then administered over $5 \mathrm{~min}$ followed by $2400 \mathrm{mg} \mathrm{m}^{-2} 5$-flurouracil IV infusion over $46 \mathrm{~h}$. For the LV5FU2 regimen, irinotecan was omitted and the 5-fluourouracil IV infusion dose was increased to $2800 \mathrm{mg} \mathrm{m}^{-2}$. There were three different FOLFOXIRI regimens, which were prescribed based on the patient's age and WHO PS status. The regimen for patients aged 70 years or less and with $\mathrm{PS}=0-1$ contained $180 \mathrm{mg} \mathrm{m}^{-2}$ irinotecan and $85 \mathrm{mg} \mathrm{m}^{-2}$ oxaliplatin, $400 \mathrm{mg} \mathrm{m}^{-2}$ 5-fluorouracil bolus and $2400 \mathrm{mg} \mathrm{m}^{-2}$ 5 -fluorouracil infusion. The individual components were reduced to $80 \%$ of full dose for patients $\geqslant 70$ years or PS $=2$ and to $60 \%$ for patients $\geqslant 70$ years and $\mathrm{PS}=2$. In arm $\mathrm{D}$, cetuximab was administered before chemotherapy as an IV dose of $500 \mathrm{mg} \mathrm{m}^{-2}$,

Table 1. Distribution of KRAS/BRAF and Topo-1 status

Topo-1 status

\begin{tabular}{|c|c|c|c|c|}
\hline \multirow[b]{2}{*}{ KRAS/BRAF status } & \multicolumn{3}{|c|}{ Topo-1 status } & \multirow[b]{2}{*}{ Total } \\
\hline & High (2-3) & Low $(0-1)$ & No result ${ }^{a}$ & \\
\hline Either mutation & $107(78)$ & $29(23)$ & $7(4)$ & $143(105)$ \\
\hline Both wild type/one wild type, other inconclusive & 135 (109) & $33(23)$ & $8(5)$ & $176(137)$ \\
\hline No result ${ }^{a}$ & $2(2)$ & $0(0)$ & $11(0)$ & $13(2)$ \\
\hline Total & 244 (189) & $62(46)$ & $26(9)$ & $332(244)$ \\
\hline \multicolumn{5}{|c|}{$\begin{array}{l}\text { Abbreviation: Topo- } 1=\text { topoisomerase } 1 . \\
{ }^{2} \text { No result due to either inconclusive result or missing or failed test. } \\
\text { Tests of association (for those with a test result only } y \text {-bold entries in the table): Registered patients }(n=304) \text { : Pearson } \chi^{2} \text { on } 1 \text { d.f. }=0.13 ; P=0.72 \text {. Randomised patients }(n=233) \text { : Pearson } \chi^{2} \text { on } 1 \\
\text { d.f. }=1.03 ; P=0.31 \text {. Numbers are: } N \text { registered ( } N \text { randomised). }\end{array}$} \\
\hline
\end{tabular}


whereas in arm E bevacizumab was administered first as a $5 \mathrm{mg} \mathrm{kg}^{-1}$ IV infusion. All of the regimens are described in detail in the FOCUS 3 protocol (Maughan and Meade, 2010).

If molecular results were not confirmed by 2 weeks, patients could have one cycle of LV5FU2 before randomisation. Treatment continued for at least 24 weeks or until disease progression on treatment.

Patient symptoms were scored using National Cancer Institute Common Toxicity Criteria for Adverse Events version 3.0. SAEs and deaths, together with an assessment of causality, were continuously reported; and were reassessed by an experienced oncologist on behalf of the MRC.

CT scans were performed within 5 weeks before the start of treatment and then 12 weekly on treatment and evaluated using RECIST (v1.1) criteria. Responses were not confirmed by repeat scans and external radiological review was not undertaken.

Statistical methods. Analyses were conducted according to a predefined statistical analysis plan, which was approved by the FOCUS3 TMG before database lock (first analysed in August 2011, data updated for final analysis in May 2012).

For each of the co-primary process outcomes, an exact binomial 95\% confidence interval was calculated around the result. Exploratory analyses of the efficacy end points were planned in relation to the four hypotheses stated above (Trial Design), which in each case involved factorial analysis of two relevant molecular subgroups, as illustrated in Figure 1. Time-to-event curves for analysis of PFS were estimated using the Kaplan-Meier method.
All statistical analyses were carried out using Stata version 12 (StataCorp, College Station, TX, USA).

\section{RESULTS}

Patients. Between February 2010 and April 2011, 332 patients from 24 centres in the UK were registered for the FOCUS3 trial.

Topo-1 status was determined in 306 patients (92\%) and was highly expressed (2-3) in 244 (73\%). KRAS and BRAF status were determined in 319 patients (96\%), of whom 117 (37\%) had a KRAS mutation alone, 25 (8\%) BRAF mutation alone, $1(<1 \%)$ both mutations, 169 (53\%) were double wild type and $7(2 \%)$ had a $B R A F$ mutation but inconclusive KRAS status. No association was seen between topo-1 expression and KRAS/BRAF mutation status (Table 1).

Of patients registered, 288 were eligible for randomisation, and ultimately $244(85 \%)$ were randomised. The reasons why patients were not randomised are described in Figure 3 (Consort Diagram). The main baseline characteristics and treatment allocation of all randomised patients are shown in Table 2 (and in Supplementary Tables 1 and 2) and Figure 3. The distribution of KRAS/BRAF and Topo-1 status both at registration and randomisation is shown in Table 1.

Primary process outcomes. The two co-primary process outcome measures were not met. Of those patients randomised $180(74 \%)$ had their biomarker results within 10 w.d. of registration $(95 \%$ $\mathrm{CI}=68 \%, 79 \%)$. However, the results for 225 patients (92\%) were

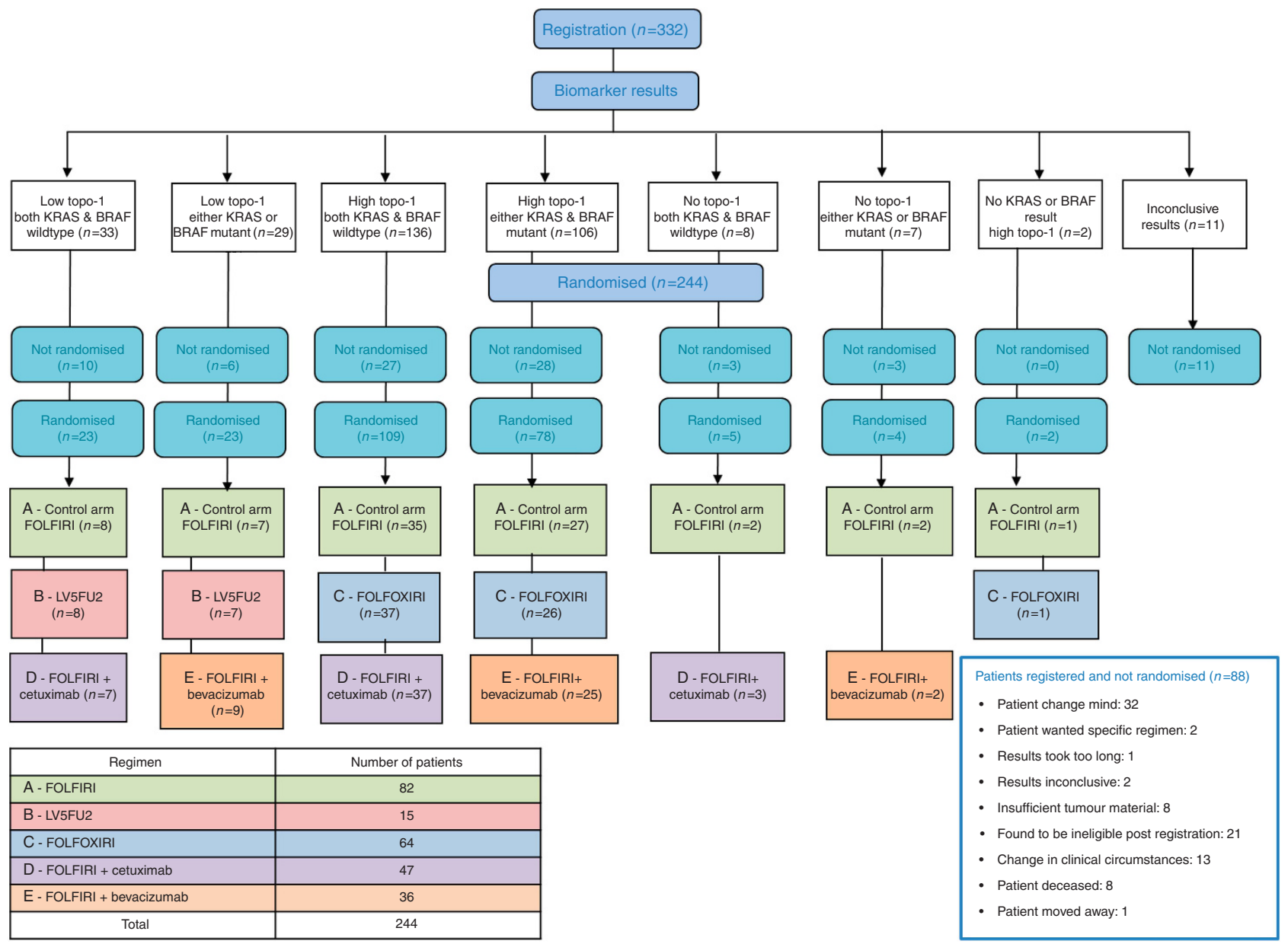

Figure 3. CONSORT diagram. 
Table 2. Baseline characteristics by treatment arm

\begin{tabular}{|c|c|c|c|c|c|c|c|c|c|c|c|c|}
\hline \multirow[b]{2}{*}{ Treatment arm } & \multicolumn{2}{|c|}{$\begin{array}{l}\text { Arm A } \\
\text { FOLFIRI }\end{array}$} & \multicolumn{2}{|c|}{$\begin{array}{c}\text { Arm B } \\
\text { LV5FU2 }\end{array}$} & \multicolumn{2}{|c|}{$\begin{array}{c}\text { Arm C } \\
\text { FOLFOXIRI }\end{array}$} & \multicolumn{2}{|c|}{$\begin{array}{c}\text { Arm D } \\
\text { FOLFIRI + cetux }\end{array}$} & \multicolumn{2}{|c|}{$\begin{array}{c}\text { Arm E } \\
\text { FOLFIRI + bev }\end{array}$} & \multicolumn{2}{|c|}{ Total } \\
\hline & $N$ & $\%$ & $N$ & $\%$ & $N$ & $\%$ & $N$ & $\%$ & $N$ & $\%$ & $N$ & $\%$ \\
\hline \multicolumn{13}{|l|}{ Sex } \\
\hline Male & 50 & 61 & 10 & 67 & 51 & 80 & 23 & 49 & 19 & 53 & 153 & 63 \\
\hline Female & 32 & 39 & 5 & 33 & 13 & 20 & 24 & 51 & 17 & 47 & 91 & 37 \\
\hline
\end{tabular}

Age at randomisation (years)

\begin{tabular}{|c|c|c|c|c|c|c|c|c|c|c|c|c|}
\hline$<45$ & 7 & 9 & 0 & 0 & 5 & 8 & 3 & 6 & 3 & 8 & 18 & 7 \\
\hline $45-54$ & 13 & 16 & 1 & 7 & 15 & 23 & 5 & 11 & 6 & 17 & 40 & 16 \\
\hline $55-64$ & 35 & 43 & 4 & 27 & 29 & 45 & 19 & 40 & 11 & 31 & 98 & 40 \\
\hline $65-74$ & 20 & 24 & 7 & 47 & 13 & 20 & 16 & 34 & 10 & 28 & 66 & 27 \\
\hline $75+$ & 7 & 9 & 3 & 20 & 2 & 3 & 4 & 9 & 6 & 17 & 22 & 9 \\
\hline
\end{tabular}

WHO performance status

\begin{tabular}{|l|c|c|c|c|c|c|c|c|c|c|c|c|}
\hline $0=$ Normal activity & 43 & 52 & 8 & 53 & 35 & 55 & 23 & 49 & 12 & 33 & 121 & 50 \\
$1=$ Restricted activity & 34 & 41 & 7 & 47 & 25 & 39 & 22 & 47 & 20 & 56 \\
$2=$ Limited self-care & 5 & 6 & 0 & 0 & 4 & 6 & 2 & 44 & 4 & 4 & 11 & 6 \\
\hline
\end{tabular}

\section{Prior radiotherapy}

\begin{tabular}{|c|c|c|c|c|c|c|c|c|c|c|c|c|}
\hline No & 75 & 91 & 15 & 100 & 64 & 100 & 45 & 96 & 34 & 94 & 233 & 95 \\
\hline Yes & 6 & 7 & 0 & 0 & 0 & 0 & 2 & 4 & 1 & 3 & 9 & 4 \\
\hline Missing & 1 & 1 & 0 & 0 & 0 & 0 & 0 & 0 & 1 & 3 & 2 & 1 \\
\hline
\end{tabular}

Adjuvant chemo

\begin{tabular}{|c|c|c|c|c|c|c|c|c|c|c|c|c|}
\hline No & 67 & 82 & 12 & 80 & 51 & 80 & 34 & 72 & 31 & 86 & 195 & 80 \\
\hline Yes, 1-6 months ago & 2 & 2 & 0 & 0 & 1 & 2 & 2 & 4 & 1 & 3 & 6 & 2 \\
\hline Yes, $>6$ months ago & 13 & 16 & 3 & 20 & 12 & 19 & 11 & 23 & 4 & 11 & 43 & 18 \\
\hline Total & 82 & 100 & 15 & 100 & 64 & 100 & 47 & 100 & 36 & 100 & 244 & 100 \\
\hline
\end{tabular}

Abbreviations: FOLFOXIRI $=$ FOLFIRI and oxaliplatin; $W H O=$ World Health Organization .

available to investigators within 15 w.d. of randomisation (95\% $\mathrm{CI}=88 \%, 95 \%)$. The interval between registration and randomisation was less than or equal to 10 w.d. in only $70(29 \%)$ patients $(95 \% \mathrm{CI}=23 \%, 35 \%)$, which suggests delays due to clinical issues (such as visit scheduling after results were available) had a greater impact on timelines than delays in biomarker analysis (Supplementary Table 3).

Reproducibility of biomarker results. 100\% concordance was achieved in the DNA mutation analysis results obtained between the two reference laboratories. Initial crossing over of topo-1 samples between the laboratories produced consistent results, although there were a higher proportion of 'high' expressing tumours than was observed in FOCUS. The Cardiff centre was not able to fully adopt the previously validated Leeds laboratory topo-1 protocol, and early in the trial it was realised that the protocols adopted at the two centres were not giving uniformly consistent results required for trial purposes. All subsequent sample testing for $K R A S, B R A F$ and topo-1 was therefore performed at Leeds.

Patient understanding. In all, $90-95 \%$ of participants selfreported that they either fully or mostly understood all of the aspects of the trial, see Figure 4 . The areas that were least well understood were the need to wait 2 weeks before start of treatment, how treatment was allocated and what happens during treatment.

Qualitative research. In-depth, interviews with 14 randomised patients were analysed using interpretative phenomenological analysis and will be published in full elsewhere. The dominant

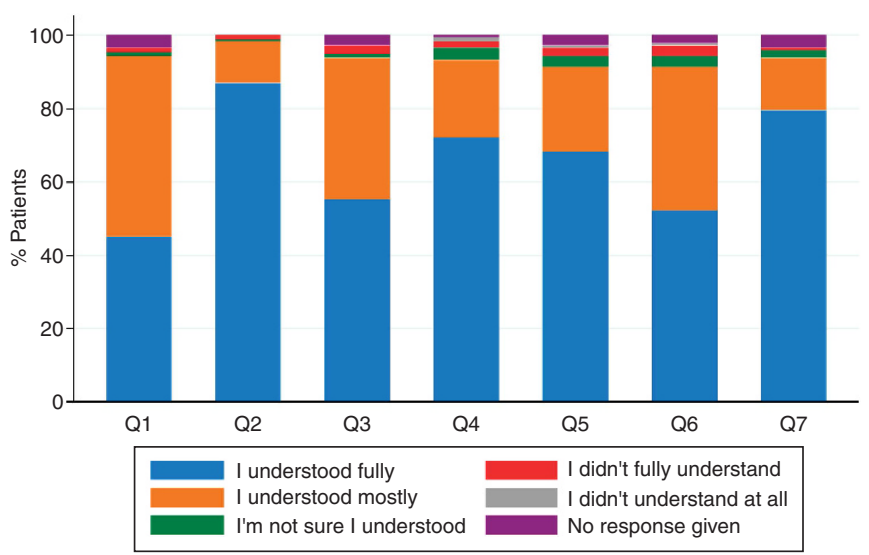

Figure 4. Patient understanding of the consent process. Q1:

Understanding of PIS2. Q2: Understanding why tumour was tested. Q3: Understanding of different treatments. Q4: Understanding of why you had to wait 2 weeks. Q5: Understanding of how treatment was allocated. Q6: Understanding of what happens during treatment. Q7: Understanding of request to give blood, complete questionnaire, take part in an interview.

issue for the majority of participants was that they were discussing the trial immediately following diagnosis of ACRC. This was a greater concern than trial entry itself. Two of the fourteen interviewees experienced delays with results from tumour testing, 
causing significant distress. The majority of patients expressed no concern with tumour testing times but highlighted distress caused by prior delays during diagnosis and treatment.

Relationships with family were key to ongoing practical and emotional support and particularly relevant to the decision to enrol on the trial and the processing of information. The multiple roles of the CRN emerged in relation to recruitment and the ongoing care of participants in the trial. Reasons for enrolling in FOCUS3 related to altruism, perception of the trial as offering personalised treatment and better care, finding a cure for cancer and being the only option available.

Treatment and follow-up. Of the 244 randomised patients, 4 did not commence treatment -2 from arm $A$ and 2 from arm E. Of the remaining 240 , two patients $(0.8 \%)$ received a single initial cycle of LV5FU2 alone before commencing their allocated regimens. Fulldose FOLFOXIRI was initiated in the $86 \%$ of patients with high topo- 1 who were $<70$ and PFS $0-1$; the remainder commenced at lower doses as per protocol. The median number of cycles of treatment delivered was $12(\mathrm{IQR}=7-13)$.

Efficacy outcomes. Efficacy outcomes were assessed in May 2012 when the median duration of follow-up was 15.2 months (IQR $=12.6-18.8$ months).

In patients with low topo-1 (B vs A, $n=30$ ), 12-week RR was $60 \%$ with LV5FU2 alone and 47\% with FOLFIRI, supporting the original hypothesis that irinotecan does not add benefit in this group. There was no evidence of a difference in PFS.

There was no improvement in RR (40\% vs $45 \%)$ or in PFS $(\mathrm{HR}=1.08(0.67-1.76))$ with the addition of oxaliplatin $(n=127)$ to FOLFIRI (C vs A). The complex randomisation algorithm resulted in a gender imbalance with more males in this group, which has uncertain relevance.

In patients with KRAS and $B R A F$ wild type (D vs $\mathrm{A}, n=92$ ), the addition of cetuximab to FOLFIRI was associated with an increased RR (44\% vs 66\%) and PFS (HR $=0.44(0.23-0.82))$, consistent with the results of the phase III Crystal trial (Van Cutsem et al, 2009, 2011).

For the addition of bevacizumab to FOLFIRI in patients with KRAS or BRAF mutations ( $\mathrm{E}$ vs $\mathrm{A}, n=72$ ), there was an observed increased RR (47\% vs 33\%). No PFS benefits were observed.

Kaplan-Meier survival curves are presented in Figure 5 and 12week RR data are summarised in Table 3.

Toxicity. Toxicity observed was as expected for the LV5FU2, FOLFIRI, FOLFIRI + cetuximab and FOLFIRI + bevacizumab regimens. The anticipated increased toxicity of the FOLFOXIRI regimen was minimal, with only $27 \%$ grade $3+$ neutropenia. This may be due to the reduced dosing schedule in the elderly/less fit patients $(n=9$ of 127$)$ previously described (Supplementary Table 4).

\section{DISCUSSION}

The primary objective of FOCUS3 was to assess the feasibility of undertaking a complex biomarker-driven trial in a national multicentre setting. Although the study did not meet either of its ambitious pre-specified co-primary process outcome measures, the trial has shown that complex prospective biomarker-driven RCTs are possible on a substantial scale across the United Kingdom. Extra resources are required in the reference pathology laboratories to undertake the biomarker analyses, but within investigator sites and the trials office there is no requirement for special dedicated staff.

Potentially eligible patients were necessarily approached for consent at precisely the time when they had recently learned of the life-threatening status of their disease; our qualitative research showed this was the dominating concern in their minds. That we achieved our target patient number from 24 centres in 1 year demonstrated that the strategy for explaining the trial was successful and that, even under difficult circumstances, complex trials can be attractive to patients. Our four-step consent procedure was developed in consultation with patients and carers and was praised by the research ethics committee. The responses to the questionnaire administered after patients had read their stage 2 PIS showed high levels of understanding of the trial. The subsequent steps in the consent process, with specific patient consent forms for each molecular cohort and for each treatment, avoided information overload and provided only that information that was specifically relevant to the particular patient.

The logistics of retrieval of the FFPE blocks from the diagnostic hospitals was a major concern. Prior written agreement, a modest (£15) fee for retrieval and detailed sample tracking by CTU personnel minimised delay. The critical lessons were the need for excellent communication between all parties in the chain: from $\mathrm{CRN}$ to pathologist to the central laboratories to the coordinating trials unit.

A delay in reporting analysis results back to the MRC CTU was observed in 22 cases and was distressing to some patients. The delays were due to insufficient tumour in the block $(n=4)$, unexpected technical difficulties $(n=6)$, initial testing inconclusive or failed $(n=12)$. This was mitigated by allowing patients $(n=2)$ to start cycle one of chemotherapy using the infusional 5-FU and folinic acid backbone, which was common to all treatment protocols and then adding in the relevant additional agents for cycle 2 once the biomarker results were available.

Overall, the most important laboratory issue was reproducibility of IHC results. Although $100 \%$ concordance was achieved in the calling of KRAS and BRAF mutations between the two laboratories, it proved very difficult to perform and report the topo-1 IHC staining intensity in a sufficiently comparable way. Owing to technical- and manpower-based organisational limitations, it was not possible to completely replicate the manual staining methodology adopted initially by the Leeds laboratory in the Cardiff laboratory where an automated staining platform was used. Even what were deemed inconsequential differences between staining protocols contributed to this lack of consistency. For future studies, contributing diagnostic centres will use the same antibodies, protocol and automated staining platform. Detailed guidance on scoring, blinded replication in contributing centres with face to face comparison of discrepantly scored sections have been implemented for IHC tests in FOCUS4. On trial quality assurance by double reading of slides will ensure comparability of evaluation.

This trial was structured so that we could address four distinct hypotheses, any or all of which might be the subject of a subsequent phase III trial. Our first hypothesis, arising from the observation in the earlier FOCUS trial that patients with low topo-1 expression appear to gain no benefit from the addition of irinotecan to LV5FU2 (Seymour et al, 2007; Braun et al, 2008), was supported and remains an intriguing one. Only 30 patients were randomised to this comparison because of the lower than expected rate of low topo-1 expression, but the high $\mathrm{RR}(60 \%)$ in the LV5FU2 only treated patients suggests further work in this area might be rewarding.

The second hypothesis proposed that patients with high topo-1 expression, who alone in FOCUS gained benefit from either irinotecan or oxaliplatin in comparison to 5-FU (Braun et al, 2008), may derive additional benefit from the triple chemotherapy regimen. With the protocol-specified dose reductions, the regimen was well tolerated. However, in contrast to the international literature (Falcone et al, 2007, 2013), although patients had a minimally higher RR, there was no hint of a PFS benefit.

The third hypothesis, tested in 92 patients with KRAS and $B R A F$ wild-type tumours, was that the addition of cetuximab 

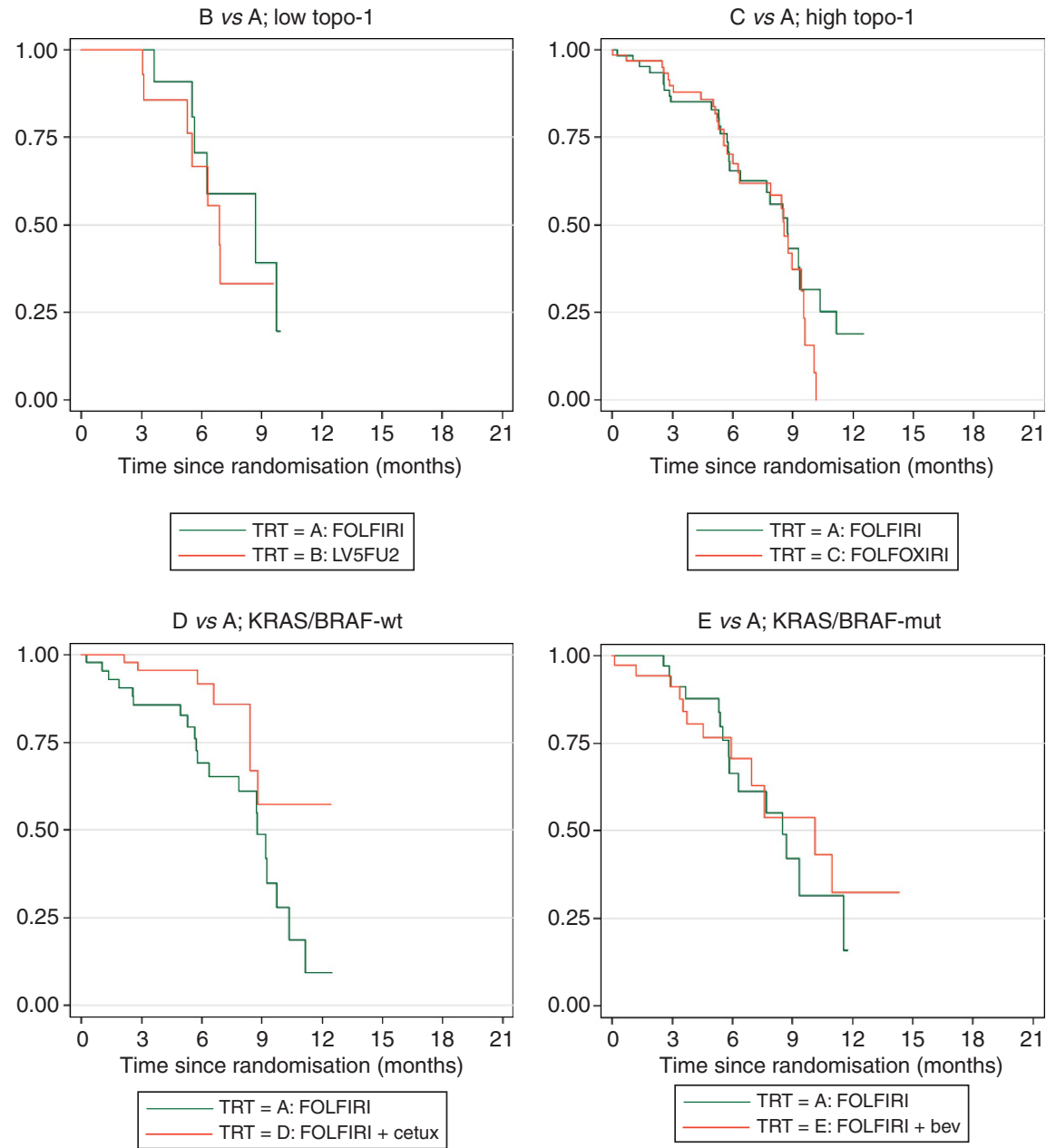

\begin{tabular}{|l|l|l|}
\hline Comparison & Model & $\mathrm{HR}(95 \% \mathrm{Cl})$ \\
\hline B vs A; low topo-1 & Unstratified & $0.84(0.38,1.86)$ \\
$(n=30)$ & Stratified & $2.18(0.56,8.51)$ \\
\hline C vs A; high topo-1 & Unstratified & $1.14(0.78,1.66)$ \\
$(n=127)$ & Stratified & $1.08(0.67,1.76)$ \\
\hline D vs A; KRAS/BRAF-wt & Unstratified & $0.77(0.49,1.21)$ \\
$(n=92)$ & Stratified & $0.44(0.23,0.82)$ \\
\hline E vs A; KRAS/BRAF-mut & Unstratified & $0.97(0.58,1.61)$ \\
$(n=72)$ & Stratified & $0.84(0.43,1.64)$ \\
\hline
\end{tabular}

Figure 5. Treatment comparisons - progression-free survival.

would increase efficacy. This recapitulated the Crystal study (Van Cutsem et al, 2009, 2011) and benefits in PFS and RR were observed.

Finally, our fourth hypothesis for patients with KRAS or BRAF mutations (72 patients) was based on the limited data that bevacizumab retains efficacy in these patients (Ince et al, 2005). No benefits on either RR or PFS were observed.

The FOCUS4 trial programme (Kaplan et al, 2013) has recently opened to recruitment building on many of the lessons learned in FOCUS3. Patient and clinician enthusiasm for biomarker-stratified trials and the rapid accrual observed in FOCUS3 have encouraged us to be optimistic in our predicted recruitment targets: 2400 registered patients with over 1500 randomised into multiple biomarker-directed comparisons in 4 years for FOCUS4. Staged PISs have been designed with information given at the time of registration limited to that which is necessary for consent for release of tumour blocks, plus a minimal outline of the protocol so as to avoid information overload. Detailed quality assurance work has been undertaken between the two biomarker reference laboratories, especially for the IHC tests (PTEN and mismatch repair proteins). In FOCUS4, the allocation by biomarker to specific comparisons occurs for patients with stable or responding disease after 4 months of first-line chemotherapy. Knowing that in FOCUS3 we completed biomarker analysis in $99 \%$ of patients within 20 w.d. of consent, the FOCUS4 logistics (registration of patients up to 12 weeks into their first-line chemotherapy) should facilitate accrual. Detailed engagement with pathologists in referring hospitals and a relatively small $(£ 15)$ payment per case enabled rapid release of blocks for central analysis in FOCUS3 and 


\begin{tabular}{|c|c|c|c|c|c|c|c|c|c|c|c|c|c|c|c|c|}
\hline \multirow{3}{*}{$\begin{array}{l}\text { Molecular subgroup } \\
\text { Treatment regimen } \\
\text { RECIST assessment }\end{array}$} & \multicolumn{4}{|c|}{ Low Topo-1 } & \multicolumn{4}{|c|}{ High Topo-1 } & \multicolumn{4}{|c|}{ KRAS/BRAF-wt } & \multicolumn{4}{|c|}{ KRAS/BRAF-mut } \\
\hline & \multicolumn{2}{|c|}{$\begin{array}{c}\text { Arm A } \\
\text { FOLFIRI }\end{array}$} & \multicolumn{2}{|c|}{$\begin{array}{c}\text { Arm B } \\
\text { LV5FU2 }\end{array}$} & \multicolumn{2}{|c|}{$\begin{array}{c}\text { Arm A } \\
\text { FOLFIRI }\end{array}$} & \multicolumn{2}{|c|}{$\begin{array}{c}\text { Arm C } \\
\text { FOLFOXIRI }\end{array}$} & \multicolumn{2}{|c|}{$\begin{array}{c}\text { Arm A } \\
\text { FOLFIRI }\end{array}$} & \multicolumn{2}{|c|}{$\begin{array}{c}\text { Arm D FOLFIRI } \\
+ \text { cetux }\end{array}$} & \multicolumn{2}{|c|}{$\begin{array}{l}\text { Arm A } \\
\text { FOLFIRI }\end{array}$} & \multicolumn{2}{|c|}{$\begin{array}{c}\text { Arm E FOLFIRI } \\
+ \text { bev }\end{array}$} \\
\hline & $N$ & $\%$ & $N$ & $\%$ & $N$ & $\%$ & $N$ & $\%$ & $N$ & $\%$ & $N$ & $\%$ & $N$ & $\%$ & $N$ & $\%$ \\
\hline Complete response & 0 & 0 & 0 & 0 & 0 & 0 & 1 & 2 & 0 & 0 & 2 & 4 & 0 & 0 & 1 & 3 \\
\hline Partial response & 7 & 47 & 9 & 60 & 25 & 40 & 28 & 44 & 20 & 44 & 29 & 62 & 12 & 33 & 16 & 44 \\
\hline Stable disease & 5 & 33 & 4 & 27 & 24 & 38 & 19 & 30 & 14 & 31 & 12 & 26 & 17 & 47 & 11 & 31 \\
\hline Progressive disease & 3 & 20 & 2 & 13 & 7 & 11 & 6 & 9 & 4 & 9 & 2 & 4 & 6 & 17 & 3 & 8 \\
\hline Missing result & 0 & 0 & 0 & 0 & 0 & 0 & 0 & 0 & 0 & 2 & 1 & 2 & 0 & 0 & 0 & 0 \\
\hline No assessment & 0 & 0 & 0 & 0 & 7 & 11 & 10 & 16 & 7 & 16 & 1 & 2 & 1 & 3 & 5 & 14 \\
\hline Response rate & 7 & 47 & 9 & 60 & 25 & 40 & 29 & 45 & 20 & 44 & 31 & 66 & 12 & 33 & 17 & 47 \\
\hline $\begin{array}{l}\text { Test of association } \\
\text { (Pearson } \chi^{2} \text { on } 1 \text { d.f.) }\end{array}$ & \multicolumn{4}{|c|}{$\chi^{2}=0.54$} & \multicolumn{4}{|c|}{$\chi^{2}=0.41$} & \multicolumn{4}{|c|}{$\chi^{2}=4.31$} & \multicolumn{4}{|c|}{$\chi^{2}=1.44$} \\
\hline Total & 15 & 100 & 15 & 100 & 63 & 100 & 64 & 100 & 45 & 100 & 47 & 100 & 36 & 100 & 36 & 100 \\
\hline
\end{tabular}

the same pattern has been used in FOCUS4. Perhaps most important is the strength of the team working established through FOCUS3, including patient representatives, clinicians, biomarker experts (including histopathologists, immunohistochemists, geneticists and technicians), statisticians, research nurses, pharmacists, trial managers and data managers. To this, we have added research network managers to ensure improved patient transfers between district general hospitals and experimental cancer medicine centres, who are required in FOCUS4 for some patients randomised to the novel agent combinations being studied.

\section{CONCLUSION}

The FOCUS3 trial was a feasibility study designed to address the challenges of patient acceptability, technical logistics, and to test a novel design for examining the predictive role of biomarkers for first-line therapy of ACRC. We have shown that such studies are feasible and very well received by participants. The central trial design concepts have been taken forward into a major UK trial programme FOCUS4-molecular selection of therapy in CRC: a molecularly stratified RCT programme, which opened to accrual in January 2014 (Kaplan et al, 2013).

\section{ACKNOWLEDGEMENTS}

We are indebted to the 332 patients and their families who participated in FOCUS3.

The design of the Medical Research Council (MRC) FOCUS3 trial was conceived and developed by the National Cancer Research Institute (NCRI) advanced colorectal cancer group. The trial was funded by the MRC. Additional support was provided by Merck KGaA (free cetuximab), Pfizer and Roche (educational research grants for the MRC colorectal research portfolio). The topo-1 antibody was provided free from Leica. Laboratory work in Leeds was also supported by funding from Yorkshire Cancer Research and the Leeds Experimental Cancer Medicines Centre. All tumour samples from patients who consented for future CRC research are stored at the Wales Cancer Bank.

The MRC was the overall sponsor of the study. FOCUS3 was approved by the Medicines and Healthcare Regulatory Agency
(MHRA) on 12 June 2009 and Research Ethics Committee for Wales on 26 May 2009. The trial was coordinated by the MRC Clinical Trials Unit (CTU) following the principles of GCP, conducted with a Trial Management Group (TMG), monitored by a Data Monitoring Committee (DMC) and overseen by an independent Trial Steering Committee. Data collection at UK sites was supported by staff funding from the National Cancer Research Networks. All statistical analyses were performed at the MRC CTU. The trial is registered as an International Standard Randomised Controlled trial, number ISRCTN83171665.

Trial Management Group: TS Maughan (chair), R Adams, RH Wilson, MT Seymour, B Jasani, R Butler, S Richman, P Quirke, AM Nelson, GT Williams, G Taylor, H Grabsch, I Frayling, J Sampson, E Hodgkinson, P Rogers, M Pope and MRC CTU staff.

MRC Clinical Trials Unit: AM Meade, R Kaplan, D Fisher, SL Kenny, JK Mitchell, LL Nichols, L Harper, K Letchemanan, M Parmar.

Data Monitoring Committee: AM Meade, R Kaplan, D Fisher, TS Maughan, MT Seymour.

Trial Steering Committee: C Parker (current chair), R Rudd, J Whelan.

Sponsor: Medical Research Council.

Clinical Investigators (Institution-(number of patients contributed)): Bridgewater J, King J, Aggarwal A, Harinarayanan S, Melcher L, Karp Stephen (North Middlesex Hospital (32)), Furniss D, Wadsley J, Walkington L, Simmons T, Hornbuckle J, Pledge S, Clenton S (Weston Park Hospital (30)), Roy R, Dhadda A (Castle Hill Hospital (26)), Adams R, Maughan T, Jones R, Brewster A, Iqbal N, Arif, Crosby T (Velindre Hospital (23)), Falk S, Garadi K, Hopkins K (Bristol Haematology and Oncology Centre (18)), Seymour M, Swinson D, Anthoney A, (St James' University Hospital, Leeds (18)), Leonard P, Mohamed M, (Whittington Hospital (14)), Benstead K, Farrugia D, Shepherd S (Cheltenham General Hospital (11)), Blesing C, Hyde K, Grant W (Great Western Hospital (10)), Lowdell C, Cleator S, Riddle P, Kenny L, Ahmad R (Charing Cross Hospital (9)), Hill M, Bhattacharjee P, Sevitt T, Summers J, Shah R (Maidstone Hospital (9)), Whillis D, Nicholls A, Ireland H, Macgregor C (Raigmore Hospital (8)), Sizer B, Basu D (Essex County Hospital (7)), Dent J, Hofmann U (Huddersfield Royal Infirmary (6)), Roy R, Butt M, Iqbal M (Diana, Princess of Wales Hospital (6)), Dent J (Calderdale Royal Hospital (6)), Hickish T, Osborne R (Poole Hospital (3)), Hickish T, Astras G, Purandare L (Royal 
Bournemouth Hospital (2)), Tahir S, Srinivasan G (Broomfield Hospital (2)), Gollins S, Kodavatiganti R (Wrexham Maelor Hospital (2)), Bale C, Mullard A, Fuller C, Williams R, Stuart N (Ysbyty Gwynedd (1)), Gollins S, Neupane R (Glan Clwyd Hospital (1)), Bessell E, Potter V (Nottingham University Hospital (0)), Tsang D (Southend University Hospital (0)).

In addition to the above-named individuals, we acknowledge the contributions of a large number of clinicians, research nurses, data managers and other clinical and support staff at the participating centres.

\section{REFERENCES}

Braun MS, Richman SD, Quirke P, Daly C, Adlard JW, Elliott F, Barrett JH, Selby P, Meade AM, Stephens RJ, Parmar MK, Seymour MT (2008) Predictive biomarkers of chemotherapy efficacy in colorectal cancer: results from the UK MRC FOCUS trial. J Clin Oncol 26: 2690-2698.

Cheeseman SL, Joel SP, Chester JD, Wilson G, Dent JT, Richards FJ, Seymour MT (2002) A /'modified de Gramont/' regimen of fluorouracil, alone and with oxaliplatin, for advanced colorectal cancer. Br J Cancer 87: 393-399.

De Roock W, Claes B, Bernasconi D, De Schutter J, Biesmans B, Fountzilas G, Kalogeras KT, Kotoula V, Papamichael D, Laurent-Puig P, Penault-Llorca F, Rougier P, Vincenzi B, Santini D, Tonini G, Cappuzzo F, Frattini M, Molinari F, Saletti P, De Dosso S, Martini M, Bardelli A, Siena S, Sartore-Bianchi A, Tabernero J, Macarulla T, Di Fiore F, Gangloff AO, Ciardiello F, Pfeiffer P, Qvortrup C, Hansen TP, Van Cutsem E, Piessevaux H, Lambrechts D, Delorenzi M, Tejpar S (2010) Effects of KRAS, BRAF, NRAS, and PIK3CA mutations on the efficacy of cetuximab plus chemotherapy in chemotherapy-refractory metastatic colorectal cancer: a retrospective consortium analysis. Lancet Oncol 11: 753-762.

Douillard JY, Cunningham D, Roth AD, Navarro M, James RD, Karasek P, Jandik P, Iveson T, Carmichael J, Alakl M, Gruia G, Awad L, Rougier P (2000) Irinotecan combined with fluorouracil compared with fluorouracil alone as first-line treatment for metastatic colorectal cancer: a multicentre randomised trial. Lancet 355: 1041-1047.

Douillard JY, Oliner KS, Siena S, Tabernero J, Burkes R, Barugel M, Humblet Y, Bodoky G, Cunningham D, Jassem J, Rivera F, Kocákova I, Ruff P, Błasińska-Morawiec M, Šmakal M, Canon JL, Rother M, Williams R, Rong A, Wiezorek J, Sidhu R, Patterson SD (2013) Panitumumab-FOLFOX4 treatment and RAS mutations in colorectal cancer. N Engl J Med 369(11): 1023-1034.

Falcone A, Cremolini C, Masi G, Lonardi S, Zagonel V, Salvatore L, Trenta P, Tomasello G, Ronzoni M, Ciuffreda L, Zaniboni A, Tonini G,

Buonadonna A, Valsuani C, Chiara S, Carlomagno C, Boni C, Marcucci L, Boni L, Loupakis F (2013) FOLFOXIRI/bevacizumab (bev) versus FOLFIRI/bev as first-line treatment in unresectable metastatic colorectal cancer (mCRC) patients (pts): Results of the phase III TRIBE trial by GONO group. J Clin Oncol 31(suppl): abstr 3505.

Falcone A, Ricci S, Brunetti I, Pfanner E, Allegrini G, Barbara C, Crino L, Benedetti G, Evangelista W, Fanchini L, Cortesi E, Picone V, Vitello S, Chiara S, Granetto C, Porcile G, Fioretto L, Orlandini C, Andreuccetti M, Masi G (2007) Phase III trial of infusional fluorouracil, leucovorin, oxaliplatin, and irinotecan (FOLFOXIRI) compared with infusional fluorouracil, leucovorin, and irinotecan (FOLFIRI) as first-line treatment for metastatic colorectal cancer: the Gruppo Oncologico Nord Ovest. J Clin Oncol 25: 1670-1676.

Gerlinger M, Rowan AJ, Horswell S, Larkin J, Endesfelder D, Gronroos E, Martinez P, Matthews N, Stewart A, Tarpey P, Varela I, Phillimore B, Begum S, Mcdonald NQ, Butler A, Jones D, Raine K, Latimer C, Santos CR, Nohadani M, Eklund AC, Spencer-Dene B, Clark G, Pickering L, Stamp G, Gore M, Szallasi Z, Downward J, Futreal PA, Swanton C (2012) Intratumor heterogeneity and branched evolution revealed by multiregion sequencing. $N$ Engl J Med 366: 883-892.

Greaves M, Maley CC (2012) Clonal evolution in cancer. Nature 481: 306-313.

Ince WL, Jubb AM, Holden SN, Holmgren EB, Tobin P, Sridhar M, Hurwitz HI, Kabbinavar F, Novotny WF, Hillan KJ, Koeppen H (2005) Association of k-ras, b-raf, and p53 status with the treatment effect of bevacizumab. J Natl Cancer Inst 97: 981-989.
Kaplan R, Maughan T, Crook A, Fisher D, Wilson R, Brown L, Parmar M (2013) Evaluating many treatments and biomarkers in oncology: a new design. J Clin Oncol 31: 4562-4568.

Karapetis CS, Khambata-Ford S, Jonker DJ, O'callaghan CJ, Tu D, Tebbutt NC, Simes RJ, Chalchal H, Shapiro JD, Robitaille S, Price TJ, Shepherd L, Au HJ, Langer C, Moore MJ, Zalcberg JR (2008) K-ras mutations and benefit from cetuximab in advanced colorectal cancer. N Engl J Med 359: 1757-1765.

Leonard P, Seymour MT, James R, Hochhauser D, Ledermann JA (2002) Phase II study of irinotecan with bolus and high dose infusional 5-FU and folinic acid (modified de Gramont) for first or second line treatment of advanced or metastatic colorectal cancer. Br J Cancer 87 : 1216-1220.

Lievre A, Bachet JB, Le Corre D, Boige V, Landi B, Emile JF, Cote JF, Tomasic G, Penna C, Ducreux M, Rougier P, Penault-Llorca F, Laurent-Puig P (2006) KRAS mutation status is predictive of response to cetuximab therapy in colorectal cancer. Cancer Res 66: 3992-3995.

Maughan TS, Adams RA, Smith CG, Meade AM, Seymour MT, Wilson RH, Idziaszczyk S, Harris R, Fisher D, Kenny SL, Kay E, Mitchell JK, Madi A, Jasani B, James MD, Bridgewater J, Kennedy MJ, Claes B, Lambrechts D, Kaplan R, Cheadle JP (2011) Addition of cetuximab to oxaliplatin-based first-line combination chemotherapy for treatment of advanced colorectal cancer: results of the randomised phase 3 MRC COIN trial. Lancet 377: 2103-2114.

Maughan TS, Meade AM (2010) FOCUS 3 (CR12) protocol: a study to determine the feasibility of molecular selection of therapy using KRAS, BRAK and topo-1 in patients with metastatic or locally advanced colorectal cancer. Avialable at: http://www.ctu.mrc.ac.uk/plugins/ StudyDisplay/protocols/FOCUS\%203\%20Protocol\%20and\% 20appendices\%20v4\%200\%20Nov\%202010.pdf.

Sequist LV, Waltman BA, Dias-Santagata D, Digumarthy S, Turke AB, Fidias P, Bergethon K, Shaw AT, Gettinger S, Cosper AK, Akhavanfard S, Heist RS, Temel J, Christensen JG, Wain JC, Lynch TJ, Vernovsky K, Mark EJ, Lanuti M, Iafrate AJ, Mino-Kenudson M, Engelman JA (2011) Genotypic and histological evolution of lung cancers acquiring resistance to EGFR inhibitors. Sci Transl Med 3: 75ra26.

Seymour MT, Brown SR, Middleton G, Maughan T, Richman S, Gwyther S, Lowe C, Seligmann JF, Wadsley J, Maisey N, Chau I, Hill M, Dawson L, Falk S, O'callaghan A, Benstead K, Chambers P, Oliver A, Marshall H, Napp V, Quirke P (2013) Panitumumab and irinotecan versus irinotecan alone for patients with KRAS wild-type, fluorouracil-resistant advanced colorectal cancer (PICCOLO): a prospectively stratified randomised trial. Lancet Oncol 14: 749-759.

Seymour MT, Maughan TS, Ledermann JA, Topham C, James R, Gwyther SJ, Smith DB, Shepherd S, Maraveyas A, Ferry DR, Meade AM, Thompson L, Griffiths GO, Parmar MK, Stephens RJ (2007) Different strategies of sequential and combination chemotherapy for patients with poor prognosis advanced colorectal cancer (MRC FOCUS): a randomised controlled trial. Lancet 370: 143-152.

Smith JA, Osborn M (2003) Interpretative phenomenological analysis. In: Qualitative Psychology. A Practical Guide to Research Methods, Smith JA (ed), pp 51-80. Sage: London.

The Cancer Genome Network Atlas (2012) Comprehensive molecular characterization of human colon and rectal cancer. Nature 487: 330-337. Van Cutsem E, Köhne CH, Láng I, Folprecht G, Nowacki M, Cascinu S, Shchepotin I, Maurel J, Cunningham D, Tejpar S, Schlichting M, Zubel A, Celik I, Rougier P, Ciardiello F (2011) Cetuximab plus irinotecan, fluorouracil, and leucovorin as first-line treatment for metastatic colorectal cancer: updated analysis of overall survival according to tumor KRAS and BRAF mutation status. J Clin Oncol 29: 2011-2019.

Van Cutsem E, Kohne CH, Hitre E, Zaluski J, Chang Chien CR, Makhson A, D'haens G, Pinter T, Lim R, Bodoky G, Roh JK, Folprecht G, Ruff P, Stroh C, Tejpar S, Schlichting M, Nippgen J, Rougier P (2009) Cetuximab and chemotherapy as initial treatment for metastatic colorectal cancer. N Engl J Med 360: 1408-1417.

This work is published under the standard license to publish agreement. After 12 months the work will become freely available and the license terms will switch to a Creative Commons AttributionNonCommercial-Share Alike 3.0 Unported License.

Supplementary Information accompanies this paper on British Journal of Cancer website (http://www.nature.com/bjc) 